\title{
SENSIBILIDAD IN VITRO DE CEPAS DE Trichoderma AISLADAS DE SEMILLAS DE SOJA FRENTE AL FUNGICIDA MAXIM ${ }^{\circledR}$ XL
}

\author{
(In vitro sensitivity to Maxim ${ }^{\circledR}$ XL fungicide in strains of Trichoderma \\ isolated from soybean seeds)
}

Estela Durán, Marta Yasem de Romero, Eduardo Romero \& Juan Ramallo.

Cátedra de Fitopatología. Facultad de Agronomía y Zootecnia. Universidad Nacional de Tucumán, Tucumán, Argentina. ${ }^{*}$ Corresponding author. Mailing address: Cátedra de Fitopatología. Facultad de Agronomía y Zootecnia. Universidad Nacional de Tucumán. Avenida Roca 1900. (4000) Tucumán. Argentina. Tel: 54381 4330057. Fax: 543814248025 E-mail: esteladuran2003@yahoo.com.ar

Palabras clave: Trichoderma, semillas, soja, Maxim ${ }^{\circledR} \mathrm{XL}$ Key words: Trichoderma, seeds, soybean, Maxim ${ }^{\circledR} \mathrm{XL}$

\section{RESUMEN}

La utilización combinada de fungicidas a bajas dosis y cepas de Trichoderma tolerantes a los agroquímicos sería una estrategia aplicable para el manejo integrado del cultivo de soja. El objetivo del presente trabajo fue evaluar la sensibilidad in vitro de 3 cepas de Trichoderma aisladas de semillas de soja, frente a curasemilla Fludioxonil 2.5\% + Metalaxil-M 1\% (Maxim $^{\circledR}$ $X L)$. Se ensayaron 8 concentraciones de los ingredientes activos Fludioxonil 2.5\% y Metalaxil-M $1 \%$ entre los rangos $10^{-5}$ a $10^{2} \mu \mathrm{g} / \mathrm{mL}$ y $4.10^{-6}$ a $40 \mu \mathrm{g} / \mathrm{mL}$, respectivamente. El fungicida se agregó al medio Agar Papa Glucosa 2\% fundido, sembrándose un disco de agar con micelio en crecimiento activo en el centro de las placas de Petri. Se efectuaron mediciones del diámetro de las colonias cada 24 horas en 4 oportunidades. La concentración efectiva capaz de inhibir el crecimiento micelial en un $50 \%\left(C E_{50}\right)$ fue calculada mediante la función de regresión de los valores de inhibición micelial en respuesta a las diferentes concentraciones expresadas logarítmicamente. Las cepas ensayadas fueron altamente sensibles al curasemilla estudiado, con valores de $C E_{50}$ que variaron entre $8.10^{-5}$ y $1.10^{-2} \mu \mathrm{g} / \mathrm{mL}$, los que resultaron muy inferiores a la dosis agronómica del fungicida recomendada. Estos resultados indican que no sería compatible el uso conjunto del fungicida estudiado con los aislamientos de Trichoderma evaluados.

Recibido el 15 de Enero 2007

Aceptado el 2 de Abril 2007

\section{ABSTRACT}

The combined use of low fungicide doses and Trichoderma strains that are resistant to agrochemical products would constitute a viable strategy for integrated soybean cultivation management.

The aim of this work was to evaluate in vitro sensitivity to Fludioxonil 2.5\% + Metalaxil-M $1 \%$ (Maxim $\left.^{R} X L\right)$ in 3 strains of Trichoderma isolated from soybean seeds.

Eight concentrations of the active ingredient Fludioxonil $2.5 \%$ and Metalaxil-M $1 \%$ were tested, with a range of $10^{-5}$ to $10^{2} \mathrm{ug} / \mathrm{mL}$ and $4.10^{-6}$ to $40 \mathrm{ug} / \mathrm{mL}$, respectively. The fungicide was added to melted $2 \%$ Potate Glucose Agar medium, and sowing was carried out at the center of the Petri plates of an agar disk that contained fungus mycelium in active growth. Colony diameters were measured every 24 hours, on four successive occasions.

The concentration capable of inhibiting mycelial growth by $50 \%\left(C E_{50}\right)$ was calculated by means of the regression function of mycelial inhibition values obtained as response to different concentrations, which were expressed logarithmically. The three strains tested were highly sensitive to the seed treatment studied, with $C E_{50}$ values that varied between $8.10^{-5}$ and $1.10^{-2} \mathrm{ug} / \mathrm{mL}$, which are inferior to the agronomic dose recommended.

These results indicated that the joint use of the fungicide and the Trichoderma isolates evaluated would not be compatible. 


\section{INTRODUCCION}

La utilización en forma complementaria de bajas dosis de fungicidas y cepas de antagonistas tolerantes a los agroquímicos es una estrategia aplicable en el manejo integrado de los cultivos. Por este motivo, se evaluaron los efectos in vitro de diferentes fungicidas sobre la micobiota antagonista de distintos patosistemas (Kay \& Stewart, 1994; Resende et al., 2005; Santos \& Melo, 1989). Varias especies de Trichoderma han sido ampliamente reconocidas como agentes de biocontrol de enfermedades de las plantas, pero su capacidad antagónica no puede generalizarse ya que es variable según la cepa (Bettiol, 1991; Chet \& Ibar, 1991). Homechin (1987), había demostrado que concentraciones superiores a $0.1 \mathrm{ppm}$ del fungicida Captan inhibieron el crecimiento micelial de Trichoderma. Estos resultados difirieron de los obtenidos por Santos y Melo (1989) que observaron que este hongo presentó tolerancia hasta $1 \mathrm{ppm}$ de Captan. Por otro lado, Rodríguez et al. (2006), evaluaron el efecto del Carbendazim sobre el crecimiento de diferentes antagonistas aislados de muestras de suelo de lotes de soja y de otros cultivos, encontrando la mayor tolerancia al agroquímico en cepas de Talaromyces flavus y Clonostachys rosea.

Por otra parte, Mantecón (2003), al evaluar diferentes fungicidas curasemillas en el control del tizón de la vaina y del tallo (Diaporthe/Phomopsis) y «damping-off» (Fusarium spp.) en semillas de soja, encontró que Fludioxonil 2.5\%+Metalaxil 1\% (Maxim $\left.{ }^{\circledR} \mathrm{XL}\right)$ fue el más eficiente en el control de dichas enfermedades. Este producto es un fungicida de amplio espectro, sistémico y de contacto, usado habitualmente en semillas de soja (dosis de uso: $100-200 \mathrm{~mL} / 100 \mathrm{Kg}$, recomendada por la Guía de Productos Fitosanitarios (2005) de la República Argentina para el control de enfermedades causadas, entre otros, por Cercospora kikuchii y especies de Fusarium, Alternaria, Phomopsis, Rhizopus, Cladosporium, Phytophtora y Pythium.

En ensayos en invernáculo, Resende et al. (2004), observaron que el fungicida Maxim no causó efectos tóxicos sobre Trichoderma y éste estaba presente en las raíces de las plantas de maíz provenientes de semillas tratadas con dicho agroquímico. Sin embargo, posteriormente Resende et al. (2005), verificaron en bioensayos que el micelio de Trichoderma harzianum que permaneció en contacto directo con el fungicida Maxim resultó con un menor índice de crecimiento micelial.

El objetivo de este trabajo fue evaluar la sensibilidad in vitro de 3 aislamientos de Trichoderma al fungicida Fludioxonil 2,5\% + Metalaxil-M 1\% (Maxim $\left.{ }^{\mathrm{R}} \mathrm{XL}\right)$, con el propósito de valorar la factibilidad del uso combinado del antagonista con dosis más bajas que las habitualmente recomendadas para el citado agroquímico, en sistemas de manejo integrado en soja.

\section{MATERIALES Y METODOS}

Se evaluaron tres cepas de Trichoderma (designadas TA, TB, y TC), aisladas de semillas de soja provenientes de campos comerciales de la provincia de Tucumán, las que fueron seleccionadas por su efectiva acción antagónica in vitro (Yasem et al., 2003). Para estudiar la acción inhibitoria del fungicida sobre el crecimiento de las 3 cepas de Trichoderma, se ensayaron 8 concentraciones del compuesto comercial Maxim ${ }^{\circledR} \mathrm{XL}$ cuyos ingredientes activos son Fludioxonil 2.5\% + Metalaxil-M 1\%, correspondiendo al primero el rango de concentraciones entre $10^{-5}$ a $10^{2} \mu \mathrm{g} / \mathrm{mL}$ y al segundo entre $4 \times 10^{-6}$ a $40 \mu \mathrm{g} / \mathrm{mL}$. La mayor concentración correspondió a la cincuentava parte de la dosis agronómica recomendada por la Guía de Productos Fitosanitarios (2005) para la República Argentina. A $9 \mathrm{~mL}$ de medio Agar Papa Glucosa 2\% (APG) a $45-50^{\circ} \mathrm{C}$ en tubos de ensayo, fue añadido $1 \mathrm{~mL}$ de la suspensión acuosa del producto en concentración diez veces mayor que las arriba mencionadas y fue transferido a placas de Petri de $9 \mathrm{~cm}$ de diámetro.

De los 3 cultivos de las cepas de Trichoderma en APG de 5 días de desarrollo, se extrajeron con un sacabocado, discos de $6 \mathrm{~mm}$ de diámetro que fueron sembrados en el centro de la placa de Petri que contenía APG más el agregado del fungicida en cada una de las diluciones descritas.

Se utilizó un diseño experimental totalmente aleatorizado con seis repeticiones por cada aislamiento (3) y por cada dilución del fungicida (8), con el control correspondiente. El testigo consistió en la siembra de discos de $6 \mathrm{~mm}$ de diámetro de cada cepa de Trichoderma ensayada en 6 placas con $9 \mathrm{~mL}$ de APG más $1 \mathrm{~mL}$ de agua destilada estéril. Las placas fueron incubadas a $26 \pm 2{ }^{\circ} \mathrm{C}$ con ciclos de $12 \mathrm{~h}$ de luz/oscuridad. Las mediciones de crecimiento micelial fueron realizadas cada 24 horas, en cuatro oportunidades sucesivas, midiendo dos diámetros de la colonia en posición ortogonal de cada placa. El porcentaje de inhibición micelial in vitro relativo al testigo de cada cepa de Trichoderma para cada dosis y réplica, fue calculado por la siguiente fórmula: 100 x [(Diámetro testigo - 6) - (Diámetro Tratamiento - 6)] / (Diámetro testigo - 6). Se determinó, según el método propuesto por Mónaco et.al (2001), la regresión lineal [ $\mathrm{Y}=\mathrm{a}+\mathrm{b}(\log \mathrm{x})]$ entre el porcentaje de inhibición relativo al testigo (Y: dato no transformado) con el $\log _{10}$ de las concentraciones evaluadas del producto $(\mathrm{x})$, incluyendo 6 réplicas por dosis, aislamiento y evaluación (24, 48, 72 y 96 h). $\mathrm{La} \mathrm{CE}_{50}$ para cada caso fue calculada como: $\mathrm{CE}_{50}=10^{(50-\mathrm{a}) / \mathrm{b}}$. 
Tabla 1. Relación entre la inhibición relativa al testigo del crecimiento de las 3 cepas de Trichoderma y la dosis del fungicida Fludioxonil $2.5 \%$ + Metalaxil M $1 \%$.

\begin{tabular}{|c|c|c|c|c|}
\hline Cepas de Trichoderma & $\begin{array}{c}\text { Evaluación } \\
\text { (horas) }\end{array}$ & Ecuación de Regresión & $\mathbf{R}^{2}$ & $\begin{array}{c}\mathbf{C E}_{50} \\
(\mu \mathrm{g} / \mathrm{ml})\end{array}$ \\
\hline Сера TA & $\begin{array}{l}24 \\
48 \\
72 \\
96\end{array}$ & $\begin{array}{l}\mathrm{Y}=19.04 * \log _{10} \mathrm{x}+128.03 \\
\mathrm{Y}=15.25 * \log _{10} \mathrm{x}+87.25 \\
\mathrm{Y}=15.95 * \log _{10} \mathrm{x}+86.07 \\
\mathrm{Y}=16.29 * \log _{10} \mathrm{x}+86.11\end{array}$ & $\begin{array}{l}0.901 * * * \\
0.885 * * * \\
0.949 * * * \\
0,936 * * *\end{array}$ & $\begin{array}{l}0.00008 \\
0.00361 \\
0.00549 \\
0.00608\end{array}$ \\
\hline Сера TB & $\begin{array}{l}24 \\
48 \\
72 \\
96\end{array}$ & $\begin{array}{l}\mathrm{Y}=19.88 * \log _{10} \mathrm{x}+108.08 \\
\mathrm{Y}=19.22 * \log _{10} \mathrm{x}+90.01 \\
\mathrm{Y}=17.73 * \log _{10} \mathrm{x}+88.00 \\
\mathrm{Y}=17.75 * \log _{10} \mathrm{x}+84.22\end{array}$ & $\begin{array}{l}0.887 * * * \\
0.932 * * * \\
0.955 * * * \\
0.936 * * *\end{array}$ & $\begin{array}{l}0.00120 \\
0.00830 \\
0.00720 \\
0.01182\end{array}$ \\
\hline Сера TC & $\begin{array}{l}24 \\
48 \\
72 \\
96\end{array}$ & $\begin{array}{l}\mathrm{Y}=20.11 * \log _{10} \mathrm{x}+105.16 \\
\mathrm{Y}=18.43 * \log _{10} \mathrm{x}+88.88 \\
\mathrm{Y}=16.88 * \log _{10} \mathrm{x}+85.86 \\
\mathrm{Y}=17.65 * \log _{10} \mathrm{x}+88.89\end{array}$ & $\begin{array}{l}0.776 * * * \\
0.882 * * * \\
0.859 * * * \\
0.839 * * *\end{array}$ & $\begin{array}{l}0.00181 \\
0.00780 \\
0.00750 \\
0.00627\end{array}$ \\
\hline
\end{tabular}

*** Significativo al $0.1 \%$.

Y: \% de inhibición relativo al testigo X: $\log _{10}$ de las concentraciones del producto $\left(\mathrm{R}^{2}\right)$ : coeficiente de determinación

Se determinó la significación estadística de cada ecuación seleccionada, considerando el coeficiente de determinación $\left(\mathrm{R}^{2}\right)$.

\section{RESULTADOS Y DISCUSION}

La Tabla 1 muestra la relación entre la inhibición del crecimiento relativa al testigo de aislamientos de Trichoderma ensayados y la dosis expresadas como $\log _{10}$ del fungicida Fludioxonil 2.5\% + Metalaxil M 1\%, en cuatro oportunidades de evaluación para las 3 cepas de Trichoderma.

En la Figura 1 se observa la inhibición de las colonias desarrolladas sobre APG con diferentes concentraciones del fungicida y la colonia testigo de Trichoderma. La Figura 2 muestra las rectas de regresión de las cepas ensayadas para el agroquímico a las 24 y 48 h. de incubación.

El coeficiente de determinación mostró baja variabilidad, no explicada por la regresión lineal, los ajustes fueron confiables y altamente significativos $\left(R^{2} \geq 0,776\right)$ y se obtuvo una buena precisión en la predicción de $\mathrm{CE}_{50} \mathrm{de}$ las cepas analizadas.

Las concentraciones crecientes ensayadas se relacionaron casi al 100\% de las variaciones del diámetro de crecimiento del hongo observadas $\left(R^{2} \geq 0,77\right)$. Si bien a las $24 \mathrm{~h}$ se determinaron valores muy bajos de $\mathrm{CE}_{50}$, TA fue más sensible que las otras dos cepas (TB y TC), las que fueron similares entre sí. Este comportamiento, aunque menos marcado, se observó también a las 48, 72 y 96 h, y sus respectivas rectas de regresión y los valores de $\mathrm{CE}_{50}$ determinados para cada cepa ensayada (Tabla 1), permitieron establecer claramente la sensibilidad al curasemilla de Trichoderma sp. in vitro. Los valores de $\mathrm{CE}_{50}$ variaron entre $8.10^{-5}$ y $1.210^{-2} \mathrm{ìg} / \mathrm{mL}$ después de las 24,48 , 72 y 96 h de incubación.

Los resultados obtenidos en el presente trabajo no concuerdan con Resende et al. (2004), pero en este caso, probablemente, los fungicidas probados no causaron efecto tóxico en Trichoderma por lixiviación del producto en el suelo. Sin embargo, coinciden con los resultados obtenidos por Homechin (1987), Resende et al. (2005), y Rodríguez et al. (2006), quienes verificaron distintos grados de incompatibilidad de Trichoderma en contacto directo con diferentes fungicidas.

En las concentraciones ensayadas, el fungicida curasemillas Fludioxonil 2.5\%+ Metalaxil M 1\% provocó una marcada inhibición del crecimiento micelial de los aislamientos de Trichoderma evaluados.

Los valores de $\mathrm{CE}_{50}$ para las cepas analizadas fueron muy inferiores a la dosis agronómica recomendada por el fabricante para la soja, por lo que no sería factible el uso combinado de los mismos en sistemas de manejo integrado en soja. 


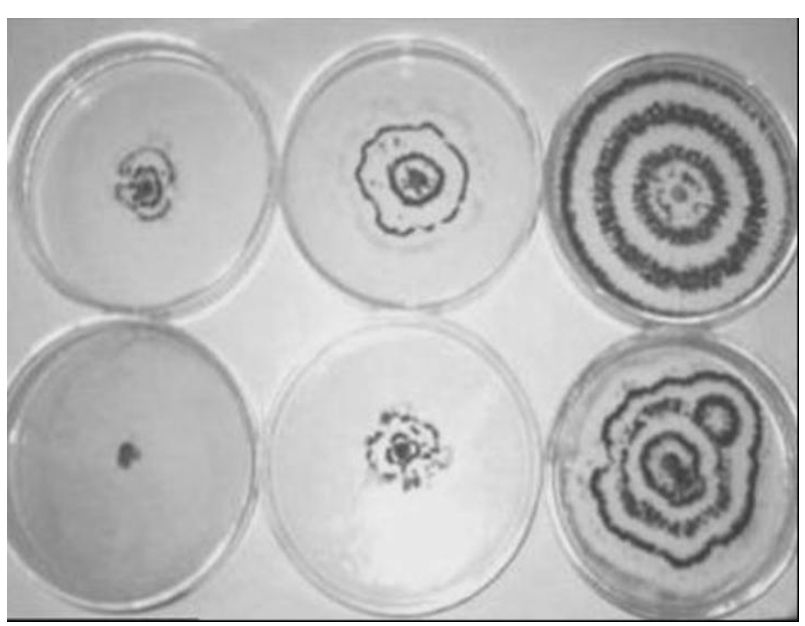

Figura 1. Colonias en APG de Trichoderma (cepa TA) con concentraciones decrecientes de Fludioxonil $2.5 \%$ + Metalaxil M 1\% (de izquierda a derecha), y del testigo, sin agroquímico (ángulo superior derecho), incubadas durante $96 \mathrm{~h}$.

\section{AGRADECIMIENTOS}

Este trabajo ha sido subsidiado por el Consejo de Investigaciones de la Universidad Nacional de Tucumán (CIUNT) Programa No. 26/A333.

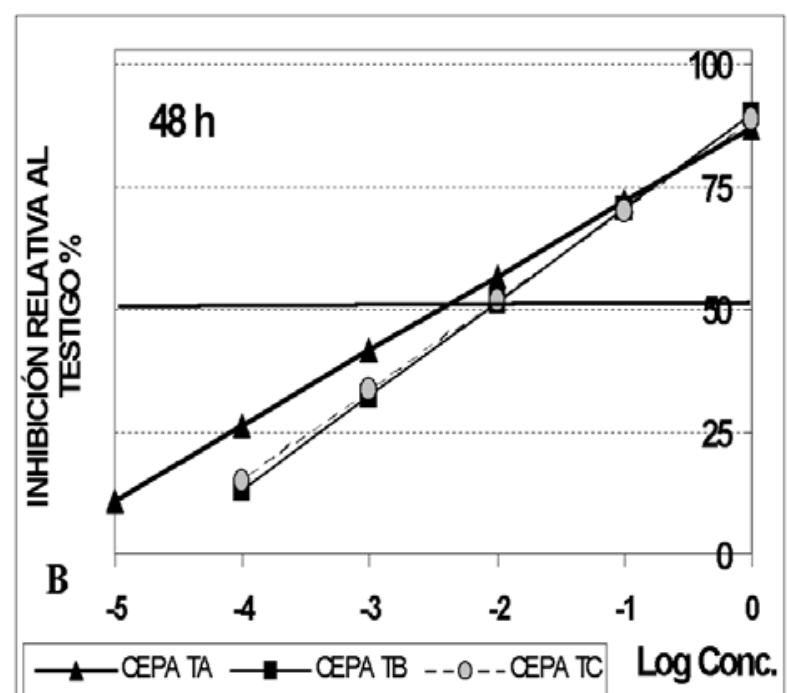

Figura 2. Regresiones determinadas entre el logaritmo de las concentraciones del Fludioxonil $2.5 \%$ + Metalaxil M $1 \%\left(10^{-5}\right.$ a $10^{2} \mu \mathrm{g} / \mathrm{mL}$ y $4.10^{-6}$ a $40 \mu \mathrm{g} / \mathrm{mL}$, respectivamente) e inhibición porcentual del crecimiento micelial de las cepas de Trichoderma en relación al testigo, a las 24 (A) y 48 hs (B) de incubación. Los valores de las concentraciones del fungicida fueron transformados en $\log _{10}$.

\section{REFERENCIAS}

Bettiol, W. (1991). Controle biológico de doenças em plantas. Jaguariúna, São Paulo, Brasil, EMBRAPA-CNPMA.

Chet, I. \& Ibar, J. (1991). Biological control of fungal pathogens. Applied Biochem. Biotechnol. 48:37-43

Guia de Productos Fitosanitarios (2005). Cámara de Sanidad Agropecuaria y Fertilizantes Republica Argentina (CASAFE) 2da. ed. Tomo 2

Homechin, M. (1987). Potencial em emprego de isolados brasileiros de Trichoderma harzianum para controle de patogenos de soja (Glicine max (L.) Merril). Tese doutorado. Escola Superior de Agricultura Luiz de Queiroz. Piracicaba, São Paulo, Brasil.

Kay, S.J. \& Stewart, A. (1994). The effect of fungicides on fungal antagonists of onion white rot and selection of dicarboximide-resitant biotypes. Plant Pathology 43:863-871

Mantecón, J.D. (2003). Evaluación de fungicidas curasemillas en el control del tizón de la vaina y del tallo (Diaporthe/Phomopsis) y damping-off (Fusarium spp.) en semillas de soja. http:// www.inta.gov.ar/balcarce/info/documentos/agric/oleag/soja/ mantecon2.htm. Instituto Nacional de Tecnología Agropecuaria (INTA).

Mónaco, C.; Nico, A.; Rollán, M. \& Urrutia, M. (2001). Efecto in vitro de dos fungicidas sobre la micoflora antagonista al tizón temprano del tomate. Invest. Agr.: Prod. Prot. Veg. 16:325-332
Resende, M.L.; Oliveira, J.A.; Guimarães, R.M.; Von Pinho, R.G.; Vieira, A. R. (2004). Inoculação de sementes de milho utilizando o Trichoderma harzianum como promotor de crescimento. Cienc. agrotec. Lavras 28:793-798

Resende, M.L.; Pereira, C.E.; Oliveira, J.A.E. \& Guimarães, R. M. (2005). Qualidade de sementes de milho (Zea mays) tratadas com fungicida e inoculadas com Trichoderma harzianum. Revista Ciencia Agronómica 36: 60-66

Rodríguez, M. A.; Cabrera, G. M. \& Godeas, A. M. (2006). Hongos del suelo: potenciales agentes de biocontrol para el manejo de enfermedades en soja. $3^{\circ}$ Congreso de Soja del Mercosur. Protección Vegetal 370-373

Santos, T.M. \& Melo, I.S. (1989). Resistência de isolados de Trichoderma spp. e Penicillium spp. a fungicidas in vitro. Agentes microbianos de controle de fungos fitopatogênicos. En EMBRAPACNPMA (Eds.), Boletín de Pesquisa, Jaguariúna, São Paulo, 5:18

Yasem de Romero, M.; Durán, E.L. \& Ramallo, J.C. (2003). Determinación preliminar de la actividad antagónica in vitro de Trichoderma spp. sobre Fusarium graminearum aislados de semillas de soja en Tucumán. XX Jornadas Científicas de la Asociación de Biología de Tucumán. Tafí del Valle, Tucumán. 229-230 